\title{
A Novel Risk Level Classification of Ischemic Heart Disease using Artificial Neural Network Technique - An Indian Case Study
}

\author{
K.Rajeswari, Dr.V.Vaithiyanathan and Dr.P.Amirtharaj
}

\begin{abstract}
Cardiovascular Diseases (CVD) comprises of a group of diseases of the heart and vascular system. The major conditions include Coronary Heart Disease (CHD) or Ischemic Heart Disease (IHD) which causes 25-30 percent of deaths in most industrialized countries. India is in a risk of developing more death due to CHD. Hence a Decision Support System (DSS) is proposed to identify the level of risk in Ischemic Heart Disease of a Patient. This will help the patients in taking precautionary steps like following a balanced diet and medication which in turn may increase the life time of a patient. The features for prediction are selected after considering Indian conditions from literature and based on the Expert knowledge from Doctors. Framingham Risk score which has five attributes is used for comparison. Our proposed system will have seventeen features to be analyzed according to Indian Conditions. The system proposes implementation of Artificial Neural Network technique to mine the knowledge from Medical data collected.
\end{abstract}

Index Terms-Decision Support System (DSS); Ischemic Heart disease (IHD); Artificial Neural Network; Coronary Heart Disease (CHD); Risk.

\section{INTRODUCTION}

When it comes to matters of the heart Indians seem to fare badly. By the end of next year, India will bear $60 \%$ of the world's heart disease burden. What is worse, compared to people in other developed countries, the average age of patients with heart disease is at least 5-8 years lower among Indians. Sixty is the average age of heart patients in India against 63-68 in developed countries. It is slipping further to the mid-50s. Indians are also more likely to have types of heart disease that lead to worse outcomes like ischemic heart disease - a condition characterized by reduced blood supply to the heart[1]. The WHO has drawn attention to the fact that CHD is our modern "epidemic". Studies say that nowadays,

Manuscript received April 1, 2011.

K.Rajeswari is with Pimpri Chinchwad College of Engineering under University of Pune in the Department of Computer Engineering. She has been the winner of Cambridge Certification for Teachers and Trainers. She is very much interested in real time research problems especially in the field of medicine.(Corresponding Author Phone: +919766909526 e-mail: raji_vivek2002@yahoo.com)

Dr.V.Vaithiyanathan is with SASTRA university, Tanjore as an Associate Dean, Research, Head of department, Information Technology, and CTS Chair. He has published many papers in International conferences and Journal. (e-mail: vaithiya_nathan@hotmail.com).

Dr.P.Amirtharaj is a cardio thoracic surgeon with Madras medical Hospital. He is also an Assistant professor in Madras Medical College, Chennai , India. He has won many awards for his research contribution. (e-mail:tripark@sify.com). sudden death during sleep has become prevalent. This is due to the lack of oxygen supply to heart.

CHD may manifest itself as any one of the following forms.

1. Angina pectoris effort

2. Myocardial Infarction

3. Irregularities of the heart

4. Cardiac failure

5. Sudden death

Myocardial Infarction is specific to CHD. Others are not and may cause confusion during diagnosis [2]. Rose [3] calculates the "incubation period" of CHD may be 10 years or more.

\section{A. Coronary Heart Disease In INDIA}

A large body of data exists on the occurrence of CHD in hospital patients. However, there are only two studies one in Chandigarh, screening patients over the age 30, by a 12-lead ECG and the other in Haryana were the prevalence is 65.4 and 47.8 per 1000 males and females respectively in urban population [4].This proposed study ise carried out in Chennai, Madras Medical College.

The pattern of CHD in India has been reported to be as follows:

- CHD appears a decade earlier compared with the age incidence in developed countries. The peak period is attained between 51-60 years.

- Males are affected more than females.

- Hypertension [9] and Diabetes account for about 40 percent of all cases.

- Heavy smoking is responsible aetologically in a good number of cases [5][6][7][8].

- Serum Cholesterol[10]

- The risk of CHD is 2-3 times higher in Diabetes than in Non-Diabetes [11].

- A family history of CHD.

- Sedentary life style [12].

- Type A individuals [13].

- Higher alcohol intake, defined as 75g [9]

\section{RisK ASSESSMENT BASED ON CLINICAL CONDITIONS AND RISK FACTOR EVALUATION}

Absolute Risk is divided into three categories namely, high, intermediate and low. Patients at high risk deserve Intensive risk reduction therapy. Patients at intermediate risk also require clinical intervention to the extent that therapy is safe and effective. Patients with low risk may be encouraged by their physicians to follow health recommendations for 
prevention of CHD. Each class of absolute risk may be expressed in quantitative terms.

TABLE I. RISK CLASSIFICATION

\begin{tabular}{|l|l|}
\hline Risk Classification & $\mathbf{1 0}$ Year Absolute Risk for CHD \\
\hline Low & $<10 \%$ \\
\hline Medium & $10-20 \%$ \\
\hline High & $>20 \%$ \\
\hline
\end{tabular}

\section{A. Framingham Risk Analysis}

Framingham heart study has five features to be entered, namely Age, Total Cholesterol, Smoker/Non smoker, Systolic Blood pressure, HDL. Points are added together to predict the 10-year risk score of Myocardial Infarction. Sample score for Age is as follows [14]. Similar scores are given to other features. The scores are summed to obtain the Risk factor

\begin{tabular}{|c|c|}
\multicolumn{1}{c}{ TABLE II. } & AGE SCORE \\
\hline Age & Points \\
\hline $20-34$ & -9 \\
\hline $35-39$ & -4 \\
\hline $40-44$ & 0 \\
\hline $45-49$ & 3 \\
\hline $50-54$ & 6 \\
\hline $55-59$ & 8 \\
\hline $60-64$ & 10 \\
\hline $65-69$ & 11 \\
\hline $70-74$ & 12 \\
\hline $75-79$ & 13 \\
\hline
\end{tabular}

\section{B. Indian Heart Risk Predicting Features}

The following features are proposed to be collected and analyzed for Indian Heart risk score prediction based on extensive study and expert opinion from doctors with respect to Indian body conditions, life style and eating habits. After discussion with cardiologists a three stage questionnaire was prepared. Diagnosis is done through data collection for each individual patient as given in tables III, IV and V.

Stage I includes Physical Examination parameters. Stage II includes Co Morbid feature collection and Stage III includes attributes about personal habits and hereditary. Table VI gives the Absolute Risk for Coronary Artery Disease.

TABLE III. STAGE ONE DIAGNOSIS

\begin{tabular}{|l|l|l|l|l|}
\hline \multicolumn{3}{|c|}{ Stage 1 } \\
\hline 1.Age & 2.Gender & 3.Menopause & 4.Height & 5.Weight \\
\hline \multicolumn{2}{|l|}{ 6.BMI } & \multicolumn{3}{|l}{ 7.WaistMeasure } \\
\hline
\end{tabular}

If Sex is Female, Pre or Post Menopause details are recorded

\begin{tabular}{|} 
TABLE IV. STAGE TWO DIAGNOSIS \\
\begin{tabular}{|l|l|l|l|l|}
\hline \multicolumn{4}{|c|}{ Stage 2} \\
\hline & & & & \\
\hline 8.SBP & 9.DBP & 10. Diabetes & 11.Cholesteral & 12. Thyroid \\
& & & & \\
\hline
\end{tabular}
\end{tabular}

SBP is Systolic Blood Pressure and DBP is Diastolic Blood Pressure.
TABLE V. STAGE THREE DIAGNOSIS

\begin{tabular}{|c|c|c|c|c|}
\hline \multicolumn{5}{|c|}{ Stage 3 } \\
\hline $\begin{array}{c}\text { 13. Personal } \\
\text { habits }\end{array}$ & $\begin{array}{c}\text { 14. Family } \\
\text { History }\end{array}$ & $\begin{array}{c}\text { 15. Genetic } \\
\text { factors }\end{array}$ & 16. Type A & $\begin{array}{c}\text { 17. Sleep } \\
\text { Disturbance }\end{array}$ \\
\hline
\end{tabular}

Based on the data collected, the immediate risk analysis is classified as No Risk, Low Risk, Medium Risk or High Risk. Table VI data is collected from experienced doctors in cardiology based on the importance of every feature collected in Tables III, IV and V. Three expert opinion is collected. Two identical opinion is taken into consideration for deriving conclusion. Varied opinion data of a patient is removed from the dataset.

TABLE VI. OUTPUT

\begin{tabular}{|l|l|c|c|}
\hline \multicolumn{4}{|c|}{ Absolute Risk for CAD } \\
\hline No Risk & Low Risk & Medium Risk & High Risk \\
\hline
\end{tabular}

\section{ARTIFICIAL INTELLIGENCE IN MEDICAL DIAGNOSIS}

Nowadays many systems in health care domain are of multi-purpose type which support various complex tasks along-with diagnosis and combine more than one AI technique [17]. Medical care could be enhanced and costs could be reduced by means of an automated medical diagnosis system[15]. The importance of the role of data mining techniques in providing better patient care and effective diagnostic capabilities by finding patterns and extracting knowledge increases with the increase in the volume of stored data [16].

\section{A. Classification of CAD Risk using Artificial Neural Networks}

An artificial neural network is the simulation of human brain. It is a supervised learning technique mainly used for Non Linear Classification. Popular Neural network algorithms include Hopfield, Multilayer perceptron, Self-Organising Map, Radial Basis Function, Adaptive Resonance Theory networks, Counter Propagation networks, Back Propagation networks etc. Our paper deals with Back propagation networks. Back Propagation Network or Feed Forward Networks is a network where there is no feedback. The information flows only in forward direction. This is a systematic method for training multilayer artificial neural network [19].The network is trained by supervised learning method. As it is a gradient descent method, it reduces the total squared error of the output computed by the net. As the work deals with realistic decision making, the system should reduce the error.

\section{B. The Mathematical Model}

When creating a functional model of the biological neuron, there are three basic components of importance. First, the synapses of the neuron are modeled as weights. The strength of the connection between an input and a neuron is noted by the value of the weight. Negative weight values reflect inhibitory connections, while positive values designate excitatory connections [18]. The next two components model the actual activity within the neuron cell. An adder sums up all the inputs modified by their respective weights. This 
activity is referred to as linear combination. Finally, an activation function controls the amplitude of the output of the neuron. An acceptable range of output is usually between 0 and 1 , or -1 and 1 . The Back propagation algorithm, in particular, adaptively changes the internal network free parameters based on external stimulus. After trained, a neural network can make predictions about the membership of every test example. MLP is trained with the Back propagation algorithm suffers from the high number of parameters that need to be tuned, like learning rate, number of neurons, momentum rate, etc. However, the motivations to select this algorithm arise after observing that they have been used to solve problems in different domains, moreover, the output can be directly used for ranking purposes [21]. The algorithm was popularized by [20] and the same has been summarized below.

1. Train the network with the given set of input patient data and obtain the output after propagating through the feed forward network.

2. Compare the result obtained with the desired output as given by expert doctors.

3. Calculate the error.

4. Adjust the weight values and repeat the process until error becomes vary negligible

The detailed algorithm is as follows

1. First apply the inputs to the network and work out the output , initial output could be anything, as the initial weights were random.

2. Work out the error. The error is Expected Value - Actual value value.

Error = Output (1-Output)(Target- Output)

The "Output(1-Output)" term is necessary in the equation because of the Sigmoid Function.

3. Change the weight. Let $\mathrm{W}+\mathrm{AB}$ be the new weight and $\mathrm{WAB}$ be the initial weight.

$\mathrm{W}+\mathrm{AB}=\mathrm{WAB}+($ ErrorB $\mathrm{x}$ OutputA $)$

We update all the weights in the output layer in this way.

4. Calculate the Errors for the hidden layer neurons. Unlike the output layer we cannot calculate these directly (because we don't have a Target), so we Back Propagate them from the output layer (hence the name of the algorithm). This is done by taking the Errors from the output neurons and running them back through the weights to get the hidden layer errors. For example if neuron A is connected $B$ and $C$ then we take the errors from $\mathrm{B}$ and $\mathrm{C}$ to generate an error for $\mathrm{A}$.

ErrorA = Output A $(1$ - Output A)(ErrorB WAB + ErrorC WAC). The factor "Output (1 - Output )" is present because of the sigmoid squashing function.

5. Having obtained the Error for the hidden layer neurons now proceed as in stage 3 to change the hidden layer weights. By repeating this method we can train a network of any number of layers

The activity of hidden layer neurons is determined by the activity of input neurons and the connecting weights between the input layer and hidden layer. The activity of the output neurons depend on the activity of the hidden layer neurons and the connecting weights between the hidden and output layers. Initial weight and bias assignments influence the performance of the net. To get the best results, initial weights and biases are assigned between -0.5 to 0.5 or between -1 to +1 . Faster learning of a Back propagation network can be obtained by Nguyen widrow initialization. Also Learning Rates should be increased to improve the performance.

\section{Proposed Work}

The database as described in Table III, IV, V is collected for 125 patients. For every patient, Expert opinion from three experienced doctors about the Risk Level namely No Risk, Low Risk, Medium Risk, High Risk is obtained. If at least opinion of two doctors is similar, then the dataset is taken for training or testing. Otherwise the dataset is neglected.

The neural network of the proposed system is shown below in figure 1 . The nodes $1,2,3 \ldots, 17$ are the input nodes in the input layer. The 17 attributes collected during diagnosis of each patient is normalized in the range of 0 to 1 .The numerical attributes can be scaled in to analog form in the range between 0 to 1 using different methods. Here we have followed Linear Data scaling [19]. The number of hidden layer is chosen as 1 and the number of hidden neurons is 2 indicated as I and II in figure 1.

Optimization method is Scaled Conjugate Gradient.

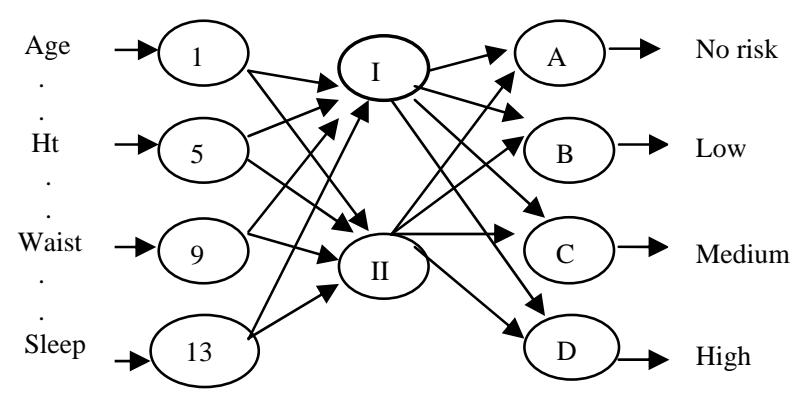

Fig 1: Neural Network Model for Ischemic Heart Disease.

\section{Results and Discussion}

After getting expert opinion, 250 patients data set is taken for analysis. 14 datasets are rejected due to lack of similarity in expert opinion. The remaining dataset of 236 patients was analyzed. The different desired classification is as in Table VI.

TABLE VI. CLASSIFICATION OF RISK

\begin{tabular}{|l|l|}
\hline Risk Cadre & No of Patients \\
\hline No Risk & 59 \\
\hline Low Risk & 51 \\
\hline Medium Risk & 69 \\
\hline High Risk & 57 \\
\hline
\end{tabular}

The tables VII, VIII and IX below show the risk level of various age groups, Gender and Sleep Disturbance. The tool PASW18 is used for analysis of all 13 input attributes towards their contribution of output. The output is denoted as No Risk, Low Risk, medium Risk and High Risk. 
TABLE VII. AgE GROUP VS RISK LEVEL MEASURE

\begin{tabular}{|c|c|c|c|c|}
\hline \multirow{2}{*}{ Age } & \multicolumn{4}{|c|}{ RiskLevel } \\
\cline { 2 - 5 } & No & Low & Medium & High \\
\hline \multirow{2}{*}{$\begin{array}{c}\text { Upto } 18 \\
19 \text { to } 35 \\
36 \text { to } 50\end{array}$} & 32 & 20 & 20 & 15 \\
\cline { 2 - 5 } & 14 & 18 & 31 & 19 \\
\cline { 2 - 5 } 51 to 65 & 2 & 7 & 14 & 18 \\
\cline { 2 - 5 } more than 65 & 0 & 0 & 1 & 2 \\
\hline
\end{tabular}

TABLE VIII. GENDER VS RISK LEVEL MEASURE

\begin{tabular}{|c|r|c|c|c|}
\hline Risk & No & Low & Medium & High \\
\hline Male & 29 & 22 & 45 & 32 \\
\hline Upto 45 & 28 & 23 & 13 & 15 \\
\hline & 2 & 6 & 11 & 10 \\
\hline
\end{tabular}

TABLE IX. SLEEPDISTURBANCE VS RISKLEVEL

\begin{tabular}{|c|c|c|c|c|c|}
\hline \multicolumn{2}{|c|}{ Cross tabulation } & \multicolumn{5}{c|}{ RiskLevel } \\
\cline { 3 - 6 } & No & Low & medium & High \\
\hline \multirow{2}{*}{$\begin{array}{c}\text { Sleep } \\
\text { Disturbance }\end{array}$} & No & 55 & 28 & 20 & 13 \\
\cline { 2 - 6 } & Yes & 4 & 23 & 49 & 44 \\
\hline
\end{tabular}

The Table $\mathrm{X}$ below shows the Average Prediction Accuracy obtained with Back Propagation Neural network.

\begin{tabular}{|c|c|} 
TABLE X. ANN PARAMETERS DURING TRAINING \\
\hline ANN Parameters & $\begin{array}{c}\text { Network } \\
\text { Architecture }\end{array}$ \\
\hline Inputs & 17 \\
\hline $\begin{array}{c}\text { Minimum Relative change in } \\
\text { Training Error }\end{array}$ & 0.0001 \\
\hline $\begin{array}{c}\text { Minimum Relative change in } \\
\text { Training Error Ratio }\end{array}$ & 0.001 \\
\hline Hidden number of units & 02 \\
\hline Initial lambda & 0.0000005 \\
\hline Initial Sigma & 0.00005 \\
\hline
\end{tabular}

Our future work will focus on reducing the number of features using optimization techniques like Genetic Algorithms; thereby will improve the Prediction Accuracy.

\begin{tabular}{|c|c|c|c|}
\multicolumn{1}{c|}{ TABLE VII. } & AVERAGE PREDICTION ACCURACY \\
\hline Method & Training & Validation & Testing \\
\hline $\begin{array}{c}\text { Back Propagation } \\
\text { Neural Network }\end{array}$ & $78 \%$ & $84 \%$ & $90 \%$ \\
\hline
\end{tabular}

Receiver Characteristic Curve graphs (ROC) shown below gives the Sensitivity vs Specificity measures of some sample attributes of our work. The sensitivity of a test is the probability that the test is positive when given to a group of patients with the disease. Specificity measures the proportion of negatives which are correctly identified.

Sensitivity $=\mathrm{TP} /(\mathrm{TP}+\mathrm{FN})$

Specificity $=\mathrm{TN} /(\mathrm{TN}+\mathrm{FP})$

TP is number of True Positives, FN is number of False Negatives.

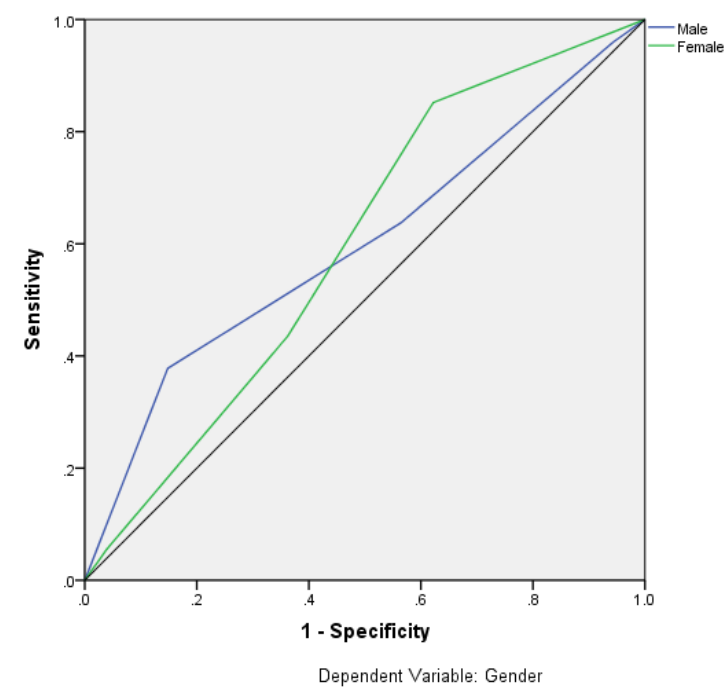

Fig 2: ROC for dependent variable Gender

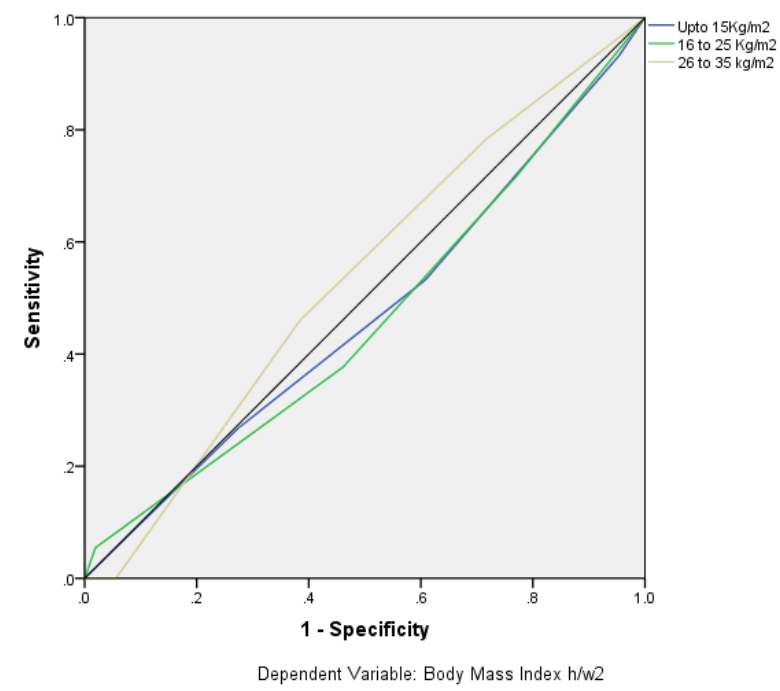

Fig 3: ROC for dependent variable BMI

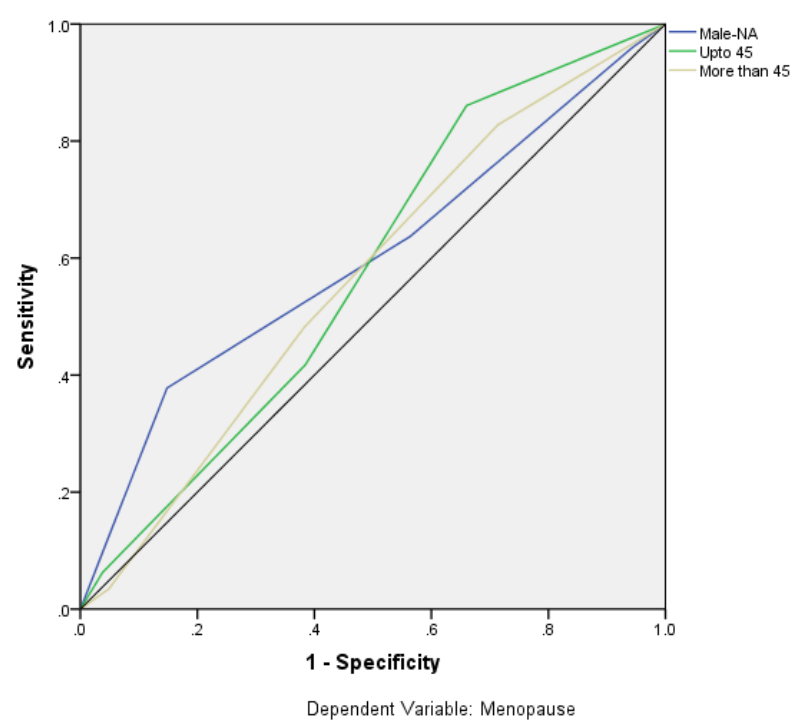

Fig 4: ROC for dependent variable Menopause 


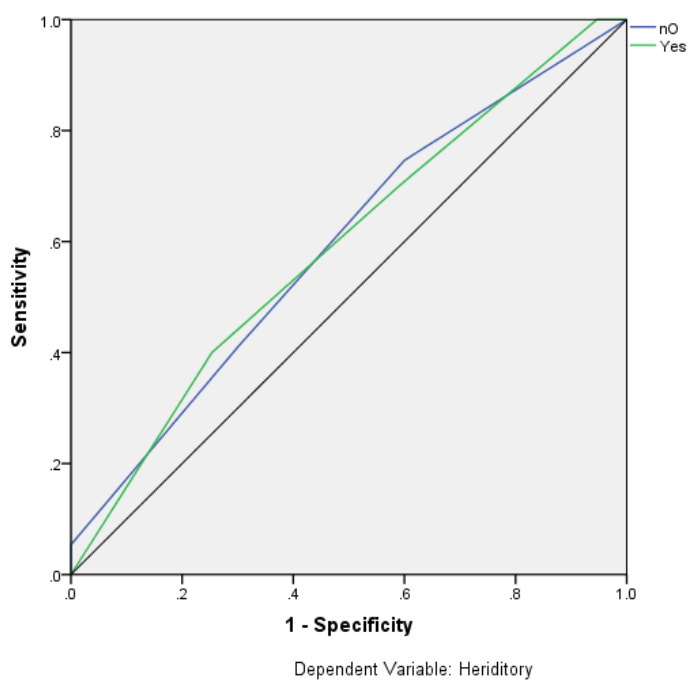

Fig 5: ROC for dependent variable Hereditary

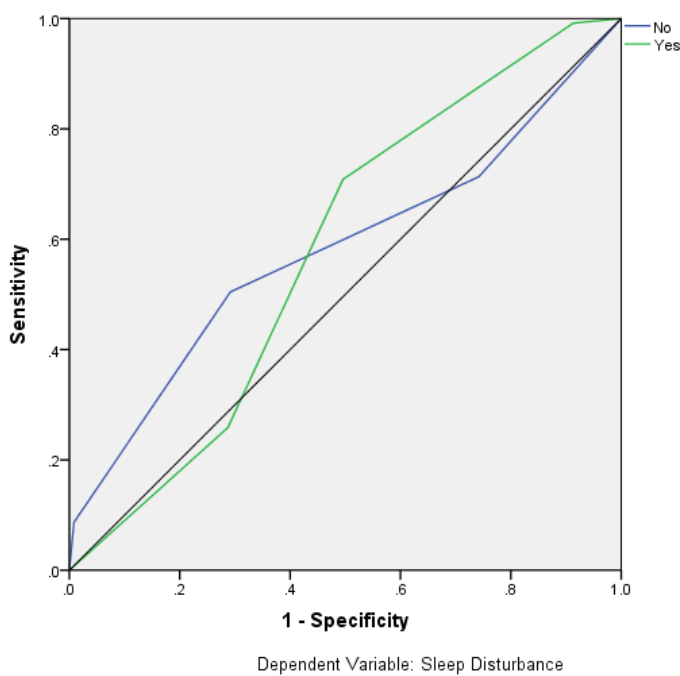

Fig 6: ROC for dependent variable sleep disturbance

The above ROC graphs show a better tradeoff between sensitivity and specificity. The idea is to develop an intelligent system, which will support a person who may be a nurse or a volunteer in a rural or urban area to make a measure of risk of IHD. This will reduce the time a Doctor has to spend in analyzing the risk a person.

The number of epochs is set to 1693. Error is 0.0326 . Learning rate and momentum are set to 0.1 and 0.2 respectively. To improve the classification process, in future work the three stages can be separately fed to three different neural networks and the results may be combined to a net.

Through this work, a patient may be directed to an expert in cardiology if the patient is found to be of High Risk or medium risk for immediate treatment or follow up procedure. A patient of low risk cadre may be suggested to have controlled food habits, smoking habits and also may be directed to meet a doctor for further guidelines. According to the Planning Commission, India is short of 600,000 doctors and 1 million nurses -for every 10,000 Indians, there is barely one doctor available.[22]. As doctors especially cardiologists are less in number we should develop a system to utilize the resource efficiently.

\section{CONCLUSION}

In this paper, we have proposed a Clinical Decision Support System (CDSS) for reliable heart disease risk classification using Artificial Intelligent techniques. The system is designed for Indian Population. Coronary Heart Disease or Ischemic Heart Disease can be handled successfully if more research is encouraged in this area. As this approach focuses on CAD Risk analysis, for a sample population, future work may be directed for further analysis.

\section{REFERENCES}

[1] http://timesofindia.indiatimes.com/city/delhi/TOI-campaign-against-h eart-disease-a- success/articleshow/7193581.cms

[2] Pedoe,H.T, “In Epidemiology of Diseases, D.I.. Miller and R.T.D. Farer(eds)”, Blackwell, Oxford, 1982.

[3] Rose,G European Heart J.3, Suppl B, 1982.

[4] Dewan BD, Malhotra KC, Gupta SP, “ Epidemiological study of coronary heart disease in rural community in Haryana”, Ind Heart J 1974; 26:68-78.

[5] Dennis Slone, M.D., Samuel Shapiro M.B., F.R.C.P. (E), Lynn Rosenberg M.S., David W. Kaufman, B.A., Stuart C. Hartz, Sc.D., Allen C. Rossi D.D.S., Paul D. Stolley, M.D., and Olli S. Miettinen, M.D.N Engl J M ed 1978; 298:1273-1276

[6] Shaper AG, Pocock SJ, Walker M, Cohen NM, Wale CJ, Thlomson AG, " British regional heart study: cardiovascular risk factors in middle-aged men in 24 towns”, BrMed3 1981;283:179-86

[7] Bain C, Hennekens CH, Rosner B, Speizer FE, “Cigarette consumption and deaths from coronary heart disease”, Jesse MJ. Lancet. 1978 May 20:1(8073):1087-8.

[8] WHO (1985). Primary Prevention of CHD EURO Rep and Studio 98. Copenhagen

[9] Keys, A, " Seven Countries : a multivariate analysis of death and CHD”, Harvard University Press, Cambridge, M.A, 1982.

[10] WHO(1985). Tech. Rep. Ser., 727.

[11] Miller, N.E., Rao,S., Lewis, B., Bjersvik, G., Myhre K. and Mjels O.D., "Highdensity lipoprotein andphysical activity”, Lancet, i (1979) 111.

[12] Jenkins CD, Rosenman RH, Zyzanski SJ, “ Prediction of clinical coronary heart disease by a test for the coronary- prone behavior pattern”, N Eng J. Med., 290:1271-1275, 1974

[13] Third Report of the National Cholestrol Education Program(NCEP) [2002].

[14] Latha, P. and Subramanian, R. (2008) "Intelligent Heart Disease Prediction System using CANFIS and Genetic Algorithm", International Journal of Biological and Medical Sciences, Vol. 3, No. 3.

[15] Wasan, S. K., Bhatnagar, V. and Kaur, H. (2006) "The Impact of Data Mining Techniques on Medical Diagnostics”, Data Science Journal, Vol. 5, pp. 119-126.

[16] Shahina B., Ahmed, M. U. and Funk, P, "Case-based systems in health sciences: a case study in the field of stress”, WSEAS Transactions on Systems, Vol. 8, No. 3, pp. 344-354,2009.

[17] Simon haykin, Neural networks, Prentice Hall 2nd edition.

[18] S.N Sivanandam, S.Sumathi, S.N.Deepa, Introduction to Neural Networks using MATLAB 6.0, Tata Mc-GrawHill Companies.

[19] D.E., Rumelhart, G.E. Hinton, and R.J. Williams, “ Learning internal representations by back-propagating errors”, Nature 323:533 - 536, 1986 (reprinted in J.A.Anderson, and E. Rosenfeld, editors, Neurocomputing.MIT Press, Cambridge, 1988).

[20] Abdi, H, “ A neural network primer. Journal of Biological Systems”, 2(3), 247-283, 1994.

[21] http://www.medicalbuyer.co.in/2007/telemecicine-an-optimistic-outlo ok-3045-41.html 